Notfall Rettungsmed 2015 · 18:447-448

DOI 10.1007/s10049-015-0061-5

Online publiziert: 2. September 2015

(c) Springer-Verlag Berlin Heidelberg 2015

CrossMark

\author{
C. Waydhas ${ }^{1} \cdot$ M. Christ ${ }^{2}$ \\ ${ }^{1}$ Chirurgische Universitätsklinik und Poliklinik Berufsgenossenschaftliches \\ Universitätsklinikum Bergmannsheil, Bochum, Deutschland \\ ${ }^{2}$ Klinikum Nürnberg Nord, Nürnberg, Deutschland
}

\title{
Ultraschalldiagnostik in der Notfallmedizin
}

nostik. Die Ultraschalldiagnostik ergänzt die klinische Untersuchung und unterstützt wichtige Differenzialdiagnosen, die für die Akutbehandlung essenziell sind, rasch zu erkennen. Allerdings sollte der Notfallmediziner das gesamte Spektrum relevanter sonographischer Untersuchungstechniken und Befundinterpretationen beherrschen, organ- und fachübergreifend. Auf der anderen Seite geht es in der Notfallmedizin nicht darum, das gesamte diagnostische Potenzial der Sonographie auszuschöpfen, sondern nur diejenigen Untersuchungsmodalitäten und Fragestellungen zu bearbeiten, die insbesondere unter den Aspekten der Lebensund Organrettung relevant sind oder auf die weiteren diagnostischen und therapeutischen Abläufe einen zeitnahen Einfluss haben können. In der Notfallmedizin ist deshalb ein breites Spektrum sonographischer Anwendungen erforderlich mit einer Fokussierung auf akut relevante Fragestellungen und unter Verzicht auf spezifische Aspekte.

Diesen Erfordernissen haben die Herausgeber versucht, Rechnung zu tragen und Beiträge zusammenzustellen, die diesen Weg weisen und dem Notfallmediziner Orientierung für den Anspruch an die eigenen Kompetenzen geben soll. Bei den Autoren handelt es sich um Kollegen, die täglich in der Notfallmedizin tätig sind, sei es in interdisziplinären Notaufnahmen, sei es stärker fachgebunden, sei es in der Rettungsmedizin. Auch bei den Reviewern der Artikel wurde größter Wert darauf gelegt, sowohl die fachspezifische als auch die generalistische Kompetenz einzubinden.

Im Themenheft werden die Rolle, der Umfang und die diagnostische bzw. therapeutische Relevanz der Ultraschalldia- gnostik für die meisten großen und notfallmedizinisch relevanten Organsysteme oder Regionen dargestellt, angefangen bei Herz, Lunge und Abdomen bis hin zum Bewegungsapparat (Beitrag der Kollegen Ackermann et al.), bei dem weniger die vital-akute Relevanz als die Vermeidung von Strahlenbelastung im Vordergrund steht. Einerseits sollen Anwendungsbereiche, wie z. B. die Lungensonographie, noch weiter als bisher als frühzeitige Routinediagnostik befördert werden (Beitrag von Glöckner et al.) oder für die Untersuchung des Abdomens (Beitrag von Görg et al.) und des Herzens (Beitrag von Schmidt et al.) den Weg weisen, welche Kompetenzen für den Notfallmediziner erforderlich oder eben verzichtbar sind.

Durch die Miniaturisierung der Technik ist es inzwischen praktikabel, tragbare Ultraschallgeräte grundsätzlich auch im Rettungsdienst einzusetzen. Wo hier mögliche Indikationen und Limitationen liegen, wird in einem eigenen Beitrag (Sacherer et al.) diskutiert. Bis hierzu abschließende Empfehlungen ausgesprochen werden können, sollten weitere Studien und Erfahrungen sowohl unter den Aspekten der Kosten-Nutzen-Abwägung als auch der Kompetenz der einzelnen Untersucher abgewartet werden.

Aber nicht nur im präklinischen Einsatz gilt die Ultraschalldiagnostik als ein stark untersucherabhängiges Verfahren. Für eine zuverlässige Durchführung und Erkennung von Befunden ist eine gute Ausbildung und Schulung unverzichtbar (Dawson et al.), um Fehldiagnosen mit möglicherweise invasiven oder nebenwirkungsbehafteten Therapiemaßnahmen zu minimieren. Auch hier ist der erforderliche Mittelweg zwischen Basisfertigkeiten und Kompetenz eines Spezialisten zu ge-
Notfallmedizin wird sie immer mehr zu einem festen Bestandteil der Notfalldiag- 


\section{Editorial}

hen. Dies kann nur im interdisziplinären Ansatz gelingen.

Die Herausgeber und Autoren hoffen, Ihnen mit diesem Themenheft einen guten Überblick über Erfordernisse und Grenzen der Notfalldiagnostik mittels Ultraschall zu geben, der Ihnen für Ihre tägliche Arbeit Anregung oder Rückhalt gibt.

Ihre

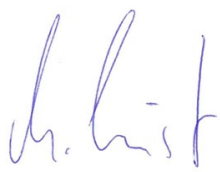

M. Christ

th. haydin

C. Waydhas

\section{Korrespondenzadresse}

Prof. Dr. C. Waydhas

Chirurgische Universitätsklinik und Poliklinik

Berufsgenossenschaftliches

Universitätsklinikum Bergmannsheil

Bürkle-de-la-Camp-Platz 1

44789 Bochum

christian.waydhas@bergmannsheil.de

Prof. Dr. M. Christ

Klinikum Nürnberg Nord

Prof.-Ernst-Nathan-Str. 1

90419 Nürnberg

Michael.Christ@klinikum-nuernberg.de 